\title{
Sensitivity analysis of periodic matrix population models*
}

\author{
Hal Caswell ${ }^{\dagger}$ \\ Biology Department, Woods Hole Oceanographic Institution \\ and \\ Max Planck Institute for Demographic Research \\ Esther Shyu \\ Biology Department, Woods Hole Oceanographic Institution
}

\begin{abstract}
Periodic matrix models are frequently used to describe cyclic temporal variation (seasonal or interannual) and to account for the operation of multiple processes (e.g., demography and dispersal) within a single projection interval. In either case, the models take the form of periodic matrix products. The perturbation analysis of periodic models must trace the effects of parameter changes, at each phase of the cycle, on output variables that are calculated over the entire cycle. Here, we apply matrix calculus to obtain the sensitivity and elasticity of scalar-, vector-, or matrix-valued output variables. We apply the method to linear models for periodic environments (including seasonal harvest models), to vec-permutation models in which individuals are classified by multiple criteria, and to nonlinear models including both immediate and delayed density dependence. The results can be used to evaluate management strategies and to study selection gradients in periodic environments.
\end{abstract}

Keywords: periodic environments; seasonal models; nonlinear models; sensitivity analysis; elasticity analysis; matrix calculus;

\section{Introduction}

Periodic matrix models are often used to study cyclical temporal variation (seasonal or interannual) or when multiple processes (e.g., demography and dispersal) operate within a single projection interval. The models take the form of periodic matrix products. A familiar example is when population projection over an annual interval is described as a product of seasonal operators. The perturbation analysis of such periodic models (Caswell and Trevisan 1994, Lesnoff et al. 2003) must specify both the vital rates affected by the perturbation and the timing of the perturbation within the cycle. In this paper, we present a general approach to the perturbation analysis of both linear and nonlinear periodic models. Our results consist of a series of formulae (summarized in Table 3) that apply directly to some of the most commonly encountered periodic models. The formulae are easily computable in a matrix-oriented language such as MATLAB or R.

If the environment is time-invariant on the scale of the projection interval (i.e., from year to year), the result is a periodic matrix population model in which the seasonal product repeats itself.

\footnotetext{
${ }^{*}$ Theoretical Population Biology (2012, in press) doi: 10.1016/j.tpb.2012.03.008

${ }^{\dagger}$ Corresponding author: Hal Caswell, Biology Department MS-34, Woods Hole Oceanographic Institution, Woods Hole, MA 02543 USA, hcaswell@whoi.edu
} 
Such a model can be written as

$$
\mathbf{n}(t+1)=\mathbf{B}_{p} \cdots \mathbf{B}_{2} \mathbf{B}_{1} \mathbf{n}(t)
$$

Here, $\mathbf{B}_{i}$ is the matrix at phase $i$ of the cycle and $p$ is the period. The period is the number of phases in the cycle; i.e., the number of matrices in the periodic matrix product in (1). The identities or the numbers of stages need not be the same from one phase to the next, so the matrices $\mathbf{B}_{i}$ may be rectangular rather than square. The phases need not be the same length, so the period may or may not be measured in units of time. For example, the model of Pico et al. (2002) contains six phases, each of 2 months duration, and the period $(p=6)$ corresponds directly to a time scale. In contrast, the model of Hunter and Caswell (2005a) has three phases, with durations about 3 weeks, 5 weeks, and 10 months, respectively. The period $(p=3)$ of the model does not correspond to a time scale, but it identifies the number of matrices in the periodic product and appears in calculations in the same role as $p=6$ in the model of Pico et al. (2002).

The projection matrix over the entire periodic cycle is ${ }^{1}$

$$
\mathbf{A}=\mathbf{B}_{p} \cdots \mathbf{B}_{2} \mathbf{B}_{1}
$$

The earliest studies of periodic matrix models were due to Darwin and Williams (1964), Skellam (1966), and MacArthur (1968). In recent years, with little fanfare, they have emerged as an important tool for incorporating multiple processes within a single projection interval. Some recent examples of their use include the following.

1. Seasonal variation. Early examples include Sarukhan and Gadgil (1974) and Lebreton and Isenmann (1976). Recent examples include studies of plants (Vavrek et al. 1997, Pico et al. 2002, Smith et al. 2005) and animals (Spencer and McGee 2001 for amphipods, Blomberg and Shine 2001 for lizards, Yoccoz et al. 1998 and Lima et al. 2003 for rodents, Lima et al. 2001 for the mouse opposum, Lesnoff et al. 2000 for a study of disease control in domestic sheep). Seasons have been variously defined in terms of monthly periods, calendar seasons, or in terms of environmental events such as rainfall or flood patterns (Lesnoff 1999, LeCorff and Horvitz 2005, Smith et al. 2005, Schleuning et al. 2008).

Although annual or near-annual species are obvious candidates for periodic models (Hyatt and Araki 2006, Ramula and Buckley 2010, Smith et al. 2005, Steets et al. 2007, Ramula 2008), within-year time scales may also be important for long-lived species. Hunter and Caswell (2005a) incorporated chick development events on a time scale of weeks into a periodic model for the sooty shearwater, which has a lifetime of decades. Jenouvrier et al. (2010) used periodic models to capture the timing of events in the breeding cycle of the emperor penguin.

\footnotetext{
${ }^{1}$ Although we will not address it in this paper, the model (1) can be written in a way that explicitly defines the starting phase in the cycle. As written, $\mathbf{A}$ in (2) projects from phase 1 to phase 1 ; if desired we could write this as $\mathbf{A}_{1}$ and define matrices

$$
\begin{aligned}
\mathbf{A}_{2} & =\mathbf{B}_{1} \mathbf{B}_{p} \cdots \mathbf{B}_{2} \\
& \vdots \\
\mathbf{A}_{p} & =\mathbf{B}_{p-1} \cdots \mathbf{B}_{1} \mathbf{B}_{p}
\end{aligned}
$$

The $\mathbf{A}_{i}$ are obtained by cyclic permutations of the sequence $\left\{\mathbf{B}_{p}, \ldots, \mathbf{B}_{1}\right\}$; each of these projects from a different phase in the cycle. Some demographic properties (e.g., the population growth rate $\lambda$ ) are invariant with respect to such permutations; others (e.g., the eigenvectors) are not (Caswell 2001). In this paper, we will start with phase 1 and refer to $\mathbf{A}$ rather than $\mathbf{A}_{1}$.
} 
2. Periodic interannual variability. Periodic models based on sequences of annual observations (e.g., Mandujano et al. 2001 for a cactus, Golubov et al. 1999 for a shrub), variations in rainfall (Mondragon et al. 2004 for an epiphyte, Vega and Montana 2004 for a grass), fire intervals (Gross et al. 1998, Hoffmann 1999, Caswell and Kaye 2001, Sinha and Brault 2005, Stokes et al. 2004), snowfall variation (Griffith et al. 2010), ENSO effects (Awkerman et al. 2006), pond levels (Ripley et al. 2004), and food availability (Gervais et al. 2006).

3. Harvest and management. These often take place at specific points within an annual or interannual cycle. Periodic models have been used to study the effects of disturbance (Pagel et al. 2008, Rydgren et al. 2001, Prevey et al. 2010), crop rotation (Davis et al. 2003, 2004, Westerman et al. 2005, Mertens et al. 2002, van den Berg et al. 2010), and harvest (e.g., Darwin and Williams 1964, Hauser et al. 2006).

4. Conditional probabilities. Periodic matrix products appear when models are written as products of conditional probabilities. In multistate mark-recapture methods, for example, a transition matrix, say $\mathbf{U}$, is written as the product of a diagonal matrix $\boldsymbol{\Sigma}$ of survival probabilities and a matrix $\mathbf{G}$ of transition probabilities conditional on survival:

$$
\mathbf{U}=\mathbf{G} \boldsymbol{\Sigma}
$$

Likelihood functions are derived in terms of the matrices $\mathbf{G}$ and $\boldsymbol{\Sigma}$ (e.g., Lebreton and Pradel 2002).

5. Multiple classifications. When individuals are classified by two or more criteria (e.g., stage and location), the dynamics over the projection interval can be described in terms of the processes affecting each criterion (e.g., transitions and movement). The result is a periodic model that uses the vec-permutation matrix to generate a block-structured projection matrix over the entire interval. This approach was introduced by Hunter and Caswell (2005b) to study classifications by stage and spatial location (see applications by Ozgul et al. 2009, Goldberg et al. 2010, Strasser et al. 2010). It has been applied to stage and environmental state (Caswell 2006, 2009b, 2011a), stage and infection status (Klepac and Caswell 2010), and stage and age (Caswell 2011b). Megamatrix models (e.g., Pascarella and Horvitz 1998, Horvitz and Tuljapurkar 2008) are a special case of the vec-permutation approach, as are block-structured multiregional matrix models (e.g., Lebreton 1996).

6. Nonlinear models. Henson and Cushing (1997) developed a model for Tribolium in an experimental system in which container size was varied periodically. Shyu et al. (in prep.) have developed a nonlinear seasonal model of an invasive plant to account for the timing of both density effects and management actions within the year. In such models, cyclic dynamics can be produced both by the environmental periodicity and the nonlinearities (e.g., Cushing 2006).

\subsection{Perturbation analysis}

The goal of perturbation analysis is to find the sensitivity or elasticity of some output variable $\boldsymbol{\xi}$ to a vector of parameters, which we will denote by $\boldsymbol{\theta}$ (Figure 1). The output variable may be a scalar (e.g., population growth rate), or a vector (e.g., the stable stage distribution), or a matrix (e.g., the fundamental matrix). The parameter vector $\boldsymbol{\theta}$ may contain matrix entries, lower-level parameters determining the matrix entries, or coefficients appearing in functions of time, density, or environmental factors. 
Table 1: Table of symbols used in the paper.

\begin{tabular}{ll} 
Symbol & Meaning \\
\hline$s_{i}$ & number of stages at phase $i$ of the cycle \\
$p$ & period of the cycle \\
$q$ & dimension of parameter vector $\boldsymbol{\theta}$ \\
$r$ & number of locations in spatial model \\
$\boldsymbol{\theta}_{i}$ & parameter vector evaluated at phase $i$ \\
$\mathbf{B}_{i}$ & projection matrix from phase $i$ to phase $i+1$, or in location $i$ \\
$\mathbf{C}_{i}^{j}$ & ordered product $\mathbf{B}_{j} \cdots \mathbf{B}_{i}$ of matrices from $i$ to $j$ \\
$\mathbf{M}_{i}$ & dispersal matrix for stage $i$ \\
$\mathbf{A}$ & projection matrix over entire cycle \\
$\mathbf{A}_{i}$ & projection matrix over cycle, starting at phase $i$ \\
$\mathbf{R}_{i}$ & matrix of LTRE contributions from phase $i$ \\
$\mathbf{E}_{s, i}$ & $s \times s$ matrix with 1 in $(i, i)$ position and 0 elsewhere \\
$\mathbf{I}_{s}$ & identity matrix of dimension $s$ \\
$\mathcal{D}(\mathbf{x})$ & diagonal matrix with $\mathbf{x}$ on the diagonal \\
$\mathbf{e}$ & vector of ones \\
$\mathbb{B}, \mathbb{M}$, etc. & block-structured matrices \\
$\circ$ & Hadamard, or element-by-element product \\
$\otimes$ & Kronecker product
\end{tabular}

In phase $i$ of the cycle, the parameter vector takes on the value $\boldsymbol{\theta}_{i}$ and determines $\mathbf{B}_{i}$. The projection matrix $\mathbf{A}$ is the periodic product of the $\mathbf{B}_{i}$. Although the output $\boldsymbol{\xi}$ is calculated from $\mathbf{A}$, the parameter dependence operates through the $\mathbf{B}_{i}$ (Figure 1). The sensitivity of $\boldsymbol{\xi}$ to the elements of $\mathbf{A}$ is in general not of interest, because those elements are complicated expressions involving the elements of all the $\mathbf{B}_{i}$, and thus mix disparate biological processes. Caswell and Trevisan (1994) introduced an way to calculate the sensitivity of population growth rate to entries of the $\mathbf{B}_{i}$, which was refined by Lesnoff et al. (2003). However, the method is limited to linear models and is not easily extended to more complicated models or to chain rule expressions.

In this paper, we present a new approach to perturbation analysis of periodic models, taking advantage of the ability of matrix calculus to compute derivatives of scalar-, vector-, or matrixvalued functions of scalar-, vector-, or matrix-valued arguments. A short introduction to matrix calculus appears in Online Supplement A. For a mathematical introduction, see Abadir and Magnus (2005); for introductions in the context of population models, see Caswell (2007, 2008), and for a complete mathematical treatment see Magnus and Neudecker 1985. Matrix calculus has been applied to a number of ecological models (Caswell 2006, 2007, 2008, 2009a,b, 2010, 2011b,c, Verdy and Caswell 2008, Klepac and Caswell 2010, Strasser et al. 2010).

We will focus on three cases that together include many of the recent examples listed above. In Section 2 we consider linear models of the form of (1) and examine a simple harvest model. In Section 3 we analyze vec-permutation models for multiple classifications. In Section 4 we analyze cyclic dynamics in a nonlinear periodic model including delayed density effects. An important application of sensitivity results is in the LTRE decomposition of effects of changes in parameters, and we present the necessary calculations in Section 5. Our results make it possible to analyze effects of changes at any point in a periodic environment. We conclude with a discussion and recommendations for implementation. 


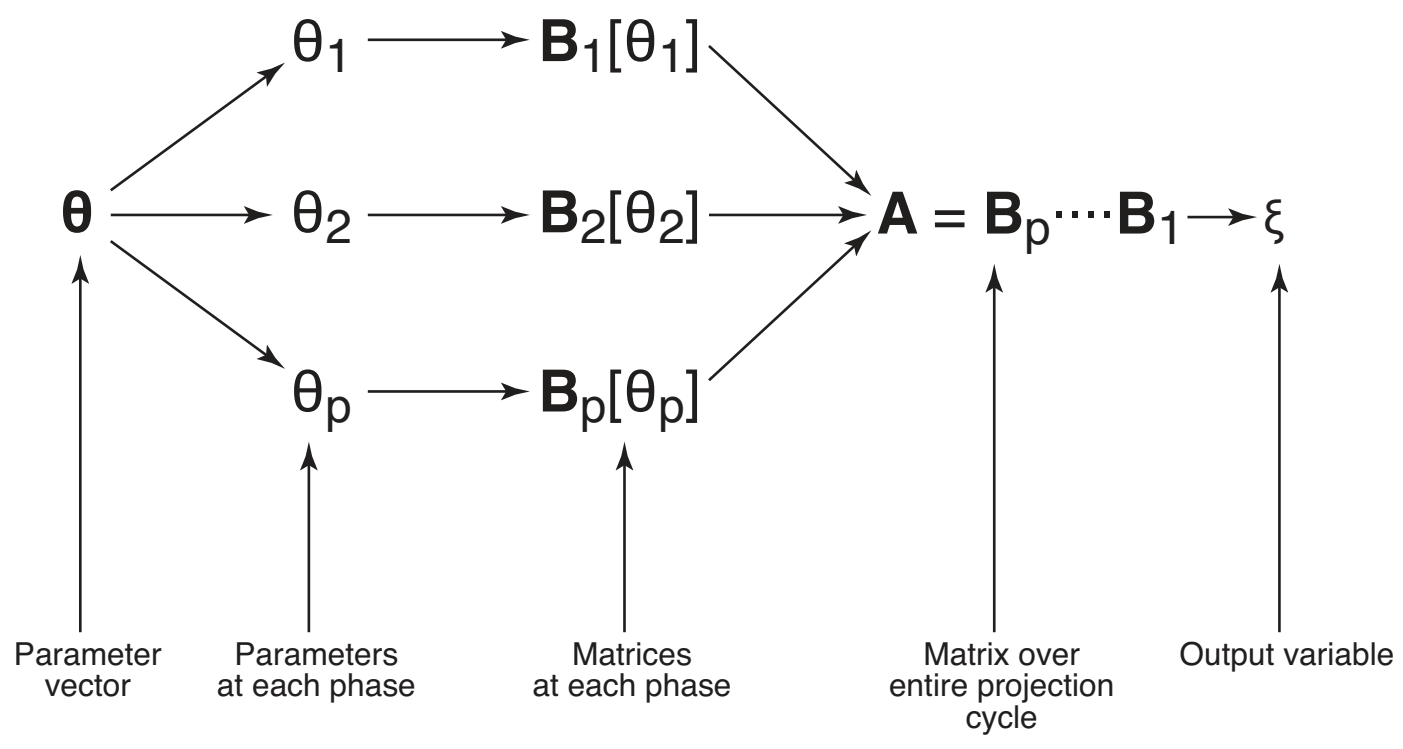

Figure 1: A vector $\boldsymbol{\theta}$ of parameters determines an output variable $\boldsymbol{\xi}$, which may be a scalar, vector, or matrix. The parameter vector will generally take on different values at each phase in the cycle, and determine the phase-specific matrix $\mathbf{B}_{i}$. These matrices determine the projection matrix $\mathbf{A}$ as a periodic matrix product; the output variable is computed from $\mathbf{A}$. The perturbation problem is to compute the sensitivity or elasticity of $\boldsymbol{\xi}$ to $\boldsymbol{\theta}$.

\section{Linear models}

Consider the basic model (1) with projection matrix (2). The period of the cycle is $p$. To allow for differences in the state vector at different phases within the cycle, define the number of stages at phase $i$ as $s_{i}$. Thus the matrix $\mathbf{B}_{i}$ is of dimension $s_{i+1} \times s_{i}$, with the subscript $i$ interpreted $\bmod (p)$ (that is, $(p+1) \bmod (p)=1)$.

Let $\boldsymbol{\xi}$ (dimension $m \times 1$ ) denote an output variable calculated from $\mathbf{A}$, where $\boldsymbol{\xi}$ might be a scalar, a vector, or a vectorized matrix. Let $\boldsymbol{\theta}(q \times 1)$ be the parameter vector. The derivative of $\boldsymbol{\xi}$ with respect to $\boldsymbol{\theta}$ is the $m \times q$ matrix

$$
\frac{d \boldsymbol{\xi}}{d \boldsymbol{\theta}^{\top}}=\left(\frac{d \xi_{i}}{d \theta_{j}}\right) \quad i=1, \ldots, m ; j=1, \ldots, q
$$

By the chain rule, all the effects of the parameters on $\boldsymbol{\xi}$ are captured in the matrix product

$$
\frac{d \boldsymbol{\xi}}{d \boldsymbol{\theta}^{\top}}=\frac{d \boldsymbol{\xi}}{d \operatorname{vec}^{\top} \mathbf{A}} \frac{d \operatorname{vec} \mathbf{A}}{d \boldsymbol{\theta}^{\top}} .
$$

The first term in (5) is the derivative of the output variable $\boldsymbol{\xi}$ with respect to the matrix $\mathbf{A}$ from which it is calculated. For example, if the dependent variable is the population growth rate $\lambda$, then

$$
\frac{d \boldsymbol{\xi}}{d \operatorname{vec}^{\top} \mathbf{A}}=\frac{d \lambda}{d \operatorname{vec}^{\top} \mathbf{A}}=\left(\mathbf{w}^{\top} \otimes \mathbf{v}^{\top}\right)
$$

where $\mathbf{w}$ and $\mathbf{v}$ are the right and left eigenvectors corresponding to $\lambda$, scaled so that $\mathbf{v}^{\top} \mathbf{w}=1$ (Caswell 2010). 
The second term in (5) is the derivative of the periodic product matrix $\mathbf{A}$ with respect to the parameter vector $\boldsymbol{\theta}$. To obtain this, differentiate (2), to obtain

$$
d \mathbf{A}=\mathbf{B}_{p} \cdots \mathbf{B}_{2} d\left(\mathbf{B}_{1}\right)+\cdots+d\left(\mathbf{B}_{p}\right) \mathbf{B}_{p-1} \cdots \mathbf{B}_{1}
$$

It is convenient to define the matrix $\mathbf{C}_{i}^{j}$ as the ordered product of the $\mathbf{B}$ matrices from $i$ up to $j$ :

$$
\mathbf{C}_{i}^{j}=\mathbf{B}_{j} \cdots \mathbf{B}_{i} \quad i \leq j
$$

and set $\mathbf{C}_{1}^{0}=\mathbf{C}_{p+1}^{p}=\mathbf{I}_{s_{1}}$. Then (7) becomes

$$
d \mathbf{A}=\mathbf{C}_{2}^{p}\left(d \mathbf{B}_{1}\right)+\mathbf{C}_{3}^{p}\left(d \mathbf{B}_{2}\right) \mathbf{C}_{1}^{1} \cdots+\left(d \mathbf{B}_{p}\right) \mathbf{C}_{1}^{p-1}
$$

Applying the vec operator to both sides gives

$$
d \operatorname{vec} \mathbf{A}=\sum_{i=1}^{p}\left[\left(\mathbf{C}_{1}^{i-1}\right)^{\top} \otimes \mathbf{C}_{i+1}^{p}\right] d \operatorname{vec} \mathbf{B}_{i}
$$

Equation (10) accounts automatically for the possibly different dimensions of the $\mathbf{B}_{i}$. Writing (10) in terms of the parameter vector $\boldsymbol{\theta}$ gives

$$
\frac{d \operatorname{vec} \mathbf{A}}{d \boldsymbol{\theta}^{\top}}=\sum_{i=1}^{p}\left[\left(\mathbf{C}_{1}^{i-1}\right)^{\top} \otimes \mathbf{C}_{i+1}^{p}\right] \frac{d \operatorname{vec} \mathbf{B}_{i}}{d \boldsymbol{\theta}^{\top}} .
$$

where $d \mathrm{vec} \mathbf{B}_{i} / d \boldsymbol{\theta}^{\top}$ is the derivative of the matrix $\mathbf{B}_{i}$ with respect to the parameter vector $\boldsymbol{\theta}$, evaluated at $\boldsymbol{\theta}_{i}$. Equation (11) sums the contributions of the derivatives of all of the phasespecific matrices $\mathbf{B}_{i}$ with respect to $\boldsymbol{\theta}$, thus accounting for all the ways in which $\boldsymbol{\theta}$ may affect the demographic rates at each point in the cycle. As written, (11) gives the result of perturbing $\boldsymbol{\theta}$ at each point in the cycle. The effect of a phase-specific perturbation is easily obtained by summing only over phases in which $\boldsymbol{\theta}_{i}$ is modified.

Substituting (11) into the formula (5) gives the general expression for the sensitivity of $\boldsymbol{\xi}$ to changes affecting any or all of the $\mathbf{B}_{i}$ :

$$
\frac{d \boldsymbol{\xi}}{d \boldsymbol{\theta}^{\top}}=\frac{d \boldsymbol{\xi}}{d \operatorname{vec}^{\top} \mathbf{A}}\left(\sum_{i=1}^{p}\left[\left(\mathbf{C}_{1}^{i-1}\right)^{\top} \otimes \mathbf{C}_{i+1}^{p}\right] \frac{d \operatorname{vec} \mathbf{B}_{i}}{d \boldsymbol{\theta}^{\top}}\right) .
$$

The elasticity of $\boldsymbol{\xi}$ can be written as follows. Let $\epsilon \boldsymbol{\xi} / \epsilon \boldsymbol{\theta}^{\top}$ be the elasticity of $\boldsymbol{\xi}$ to $\boldsymbol{\theta}$; this elasticity is the matrix

$$
\frac{\epsilon \boldsymbol{\xi}}{\epsilon \boldsymbol{\theta}^{\top}}=\left(\frac{\theta_{j}}{\xi_{i}} \frac{d \xi_{i}}{d \theta_{j}}\right)
$$

Because elasticities are logarithmic derivatives, they apply only when $\boldsymbol{\xi}>0$ and $\boldsymbol{\theta} \geq 0$. Applying this to (12) gives

$$
\frac{\epsilon \boldsymbol{\xi}}{\epsilon \boldsymbol{\theta}^{\top}}=\mathcal{D}(\boldsymbol{\xi})^{-1} \frac{d \boldsymbol{\xi}}{d \operatorname{vec}^{\top} \mathbf{A}}\left(\sum_{i=1}^{p}\left[\left(\mathbf{C}_{1}^{i-1}\right)^{\top} \otimes \mathbf{C}_{i+1}^{p}\right] \frac{d \operatorname{vec} \mathbf{B}_{i}}{d \boldsymbol{\theta}^{\top}}\right)
$$

where $\mathcal{D}(\mathbf{x})$ is a diagonal matrix with $\mathbf{x}$ on the diagonal and zeros elsewhere. 


\subsection{A simple harvest model}

The projection matrix for a simple harvest model (e.g., Hauser et al. 2006) can be written

$$
\mathbf{A}=\mathbf{B}(\mathbf{I}-\mathbf{H}) .
$$

The matrix $\mathbf{B}$ describes demography in the absence of harvest. The matrix $\mathbf{H}=\mathcal{D}(\mathbf{h})$ is a harvest matrix, where $h_{i}$ is the probability that an individual of stage $i$ is harvested. Either $\mathbf{B}$, $\mathbf{H}$, or both may be functions of a vector $\boldsymbol{\theta}$ of parameters. Differentiating (15) and applying the vec operator gives

$$
\frac{d \operatorname{vec} \mathbf{A}}{d \boldsymbol{\theta}^{\top}}=-\left(\mathbf{I}_{s} \otimes \mathbf{B}\right) \frac{d \operatorname{vec} \mathbf{H}}{d \boldsymbol{\theta}^{\top}}+\left[(\mathbf{I}-\mathbf{H})^{\top} \otimes \mathbf{I}_{s}\right] \frac{d \operatorname{vec} \mathbf{B}}{d \boldsymbol{\theta}^{\top}} .
$$

The diagonal matrix $\mathbf{H}$ can be written

$$
\mathbf{H}=\mathbf{I}_{s} \circ\left(\mathbf{h e}^{\top}\right)
$$

where $\mathbf{e}$ is a vector of ones and $\circ$ denotes the Hadamard product. Differentiating $\mathbf{H}$ in (17) gives

$$
d \operatorname{vec} \mathbf{H}=\mathcal{D}\left(\operatorname{vec} \mathbf{I}_{s}\right)\left(\mathbf{e} \otimes \mathbf{I}_{s}\right) d \mathbf{h}
$$

so that (16) becomes

$$
\frac{d \operatorname{vec} \mathbf{A}}{d \boldsymbol{\theta}^{\top}}=\underbrace{-\left(\mathbf{I}_{s} \otimes \mathbf{B}\right) \mathcal{D}\left(\operatorname{vec} \mathbf{I}_{s}\right)\left(\mathbf{e} \otimes \mathbf{I}_{s}\right) \frac{d \mathbf{h}}{d \boldsymbol{\theta}^{\top}}}_{\text {perturbations of } \mathbf{h}}+\underbrace{\left[(\mathbf{I}-\mathbf{H})^{\top} \otimes \mathbf{I}_{s}\right] \frac{d \operatorname{vec} \mathbf{B}}{d \boldsymbol{\theta}^{\top}}}_{\text {perturbations of } \mathbf{B}} .
$$

The conditional probability model (3) has the same form as the harvest model (15), so a similar analysis applies to it as well. However, the conditional transition matrix $\mathbf{G}$ is columnstochastic, because all loss of individuals is accounted for by $\boldsymbol{\Sigma}$. Thus relevant perturbations must be parameterized so that the stochasticity is preserved. For example, if $\mathbf{G}$ describes growth in the standard size-classified model (Caswell 2001 Section 4.2), e.g.,

$$
\mathbf{G}=\left(\begin{array}{ccc}
1-\gamma_{1} & 0 & 0 \\
\gamma_{1} & 1-\gamma_{2} & 0 \\
0 & \gamma_{2} & 1-\gamma_{3}
\end{array}\right)
$$

then perturbations of the $\gamma_{i}$ will preserve stochasticity of $\mathbf{G}$. If $\mathbf{G}$ has no such convenient parameterization, then changes in the entries of $\mathbf{G}$ must be compensated for by changes elsewhere in the same column (see Caswell 2001, Hill et al. 2004, and Theorem 4.5 of Caswell 2011c).

The harvest model (15) can be extended to describe harvest imposed at a specified phase within a $p$-cycle. Suppose that harvest takes place between phase $m$ and phase $m+1$, so that

$$
\mathbf{A}=\mathbf{B}_{p} \cdots \mathbf{B}_{m+1}(\mathbf{I}-\mathbf{H}) \mathbf{B}_{m} \cdots \mathbf{B}_{1}
$$

(see Darwin and Williams 1964 for an example of just this kind of seasonal harvest model). Using the same approach, it can be shown that

$$
\begin{aligned}
\frac{d \operatorname{vec} \mathbf{A}}{d \boldsymbol{\theta}^{\top}}= & -\left[\left(\mathbf{C}_{1}^{m}\right)^{\top} \otimes \mathbf{C}_{m+1}^{p}\right] \frac{d \operatorname{vec} \mathbf{H}}{d \boldsymbol{\theta}^{\top}} \\
& +\left[\mathbf{I}_{s_{1}} \otimes \mathbf{C}_{m+1}^{p}(\mathbf{I}-\mathbf{H})\right] \sum_{i=1}^{m}\left[\left(\mathbf{C}_{1}^{i-1}\right)^{\top} \otimes \mathbf{C}_{i+1}^{m}\right] \frac{d \operatorname{vec} \mathbf{B}_{i}}{d \boldsymbol{\theta}^{\top}} \\
& +\left[\left((\mathbf{I}-\mathbf{H}) \mathbf{C}_{1}^{m}\right)^{\top} \otimes \mathbf{I}_{s_{1}}\right] \sum_{i=m+1}^{p}\left[\left(\mathbf{C}_{m+1}^{i-1}\right)^{\top} \otimes \mathbf{C}_{i+1}^{p}\right] \frac{d \operatorname{vec} \mathbf{B}_{i}}{d \boldsymbol{\theta}^{\top}}
\end{aligned}
$$

The expression (18) can be substituted for $d \operatorname{vec} \mathbf{H}$ in (22), and the resulting expression for $d$ vec $\mathbf{A} / d \boldsymbol{\theta}^{\top}$ substituted into (5). 


\section{Vec-permutation models}

We consider individuals classified by stages $(1, \ldots, s)$ and locations $(1, \ldots, r)$. The population dynamics are described as if the processes acted sequentially; e.g., as if individuals would first survive and reproduce according to their demography, and then disperse among locations, and then repeat. Thus the model is formally periodic.

We will describe the analysis in terms of stages $(1, \ldots, s)$ and locations $(1, \ldots, r)$. The state of the population is given by a matrix

$$
\mathcal{N}=\left(\begin{array}{ccc}
n_{11} & \cdots & n_{1 r} \\
\vdots & & \vdots \\
n_{s 1} & \cdots & n_{s r}
\end{array}\right)
$$

where $n_{i j}$ is the number of individuals in stage $i$ at location $j$. We convert $\mathcal{N}$ into a vector for population projection using the vec operator:

$$
\mathbf{n}=\operatorname{vec} \mathcal{N}=\left(\begin{array}{c}
n_{11} \\
\vdots \\
n_{s 1} \\
\hline \vdots \\
\hline n_{1 r} \\
\vdots \\
n_{s r}
\end{array}\right)
$$

Demography in location $i$ is given by a $s \times s$ projection matrix $\mathbf{B}_{i}$. Dispersal among locations by individuals in stage $j$ is described by a $r \times r$ matrix $\mathbf{M}_{j}$. Define the block-diagonal matrices

$$
\begin{aligned}
\mathbb{B} & =\left(\begin{array}{ccc}
\mathbf{B}_{1} & \cdots & 0 \\
\vdots & \ddots & \vdots \\
0 & \cdots & \mathbf{B}_{r}
\end{array}\right) \\
& =\sum_{i=1}^{r}\left(\mathbf{E}_{s, i} \otimes \mathbf{B}_{i}\right)
\end{aligned}
$$

and

$$
\begin{aligned}
\mathbb{M} & =\left(\begin{array}{ccc}
\mathbf{M}_{1} & \cdots & 0 \\
\vdots & \ddots & \vdots \\
0 & \cdots & \mathbf{M}_{s}
\end{array}\right) \\
& =\sum_{j=1}^{s}\left(\mathbf{E}_{r, j} \otimes \mathbf{M}_{j}\right)
\end{aligned}
$$

where $\mathbf{E}_{s, i}$ is an $s \times s$ matrix containing a 1 in the $(i, i)$ position and zeros elsewhere.

Population dynamics are described as alternating between transitions among stages and movement among locations. The population is projected by

$$
\mathbf{n}(t+1)=\mathbf{K}^{\top} \mathbb{M} \mathbf{K B} \mathbf{n}(t)
$$


(Hunter and Caswell 2005b). The matrix $\mathbf{K}$ is the vec-permutation matrix, or commutation matrix, (Henderson and Searle 1981, Magnus and Neudecker 1979), which satisfies

$$
\operatorname{vec} \mathcal{N}^{\top}=\mathbf{K} \operatorname{vec} \mathcal{N}
$$

For the calculation of K, see Henderson and Searle (1981) or Hunter and Caswell (2005b).

The block-diagonal matrix $\mathbb{B}$ in (29) accounts for demographic change within locations, the vec-permutation matrix $\mathbf{K}$ rearranges the resulting vector so that the block-diagonal matrix $\mathbb{M}$ can move individuals among locations, and the matrix $\mathbf{K}^{\top}$ returns the resulting vector to its original arrangement. Thus the projection matrix is

$$
\mathbf{A}=\mathbf{K}^{\top} \mathbb{M} \mathbf{K} \mathbb{B} .
$$

The dependence of $\mathbf{A}$ on the parameters $\boldsymbol{\theta}$ can take place through $\mathbf{B}_{i}[\boldsymbol{\theta}], \mathbf{M}_{i}[\boldsymbol{\theta}]$, or both.

To apply the general formula (5), we must calculate $d \mathbf{A} / d \boldsymbol{\theta}^{\top}$. Differentiating (31) gives

$$
d \mathbf{A}=\mathbf{K}^{\top}(d \mathbb{M}) \mathbf{K} \mathbb{B}+\mathbf{K}^{\top} \mathbb{M} \mathbf{K}(d \mathbb{B}) .
$$

Applying the vec operator gives

$$
d \operatorname{vec} \mathbf{A}=\left(\mathbb{B}^{\top} \mathbf{K}^{\top} \otimes \mathbf{K}^{\top}\right) d \operatorname{vec} \mathbb{M}+\left(\mathbf{I}_{s p} \otimes \mathbf{K}^{\top} \mathbb{M} \mathbf{K}\right) d \operatorname{vec} \mathbb{B}
$$

Differentiating the block diagonal matrices (26) and (28) gives

$$
\begin{aligned}
d \mathbb{B} & =\sum_{i=1}^{r}\left(\mathbf{E}_{s, i} \otimes d \mathbf{B}_{i}\right) \\
d \mathbb{M} & =\sum_{j=1}^{s}\left(\mathbf{E}_{r, j} \otimes d \mathbf{M}_{j}\right)
\end{aligned}
$$

Applying the vec operator to both sides of (34) and (35), and using Lemma 3 of Magnus and Neudecker (1985) for the vec of a Kronecker product, gives

$$
\begin{aligned}
d \operatorname{vec} \mathbb{B} & =\sum_{i=1}^{r}\left(\mathbf{I}_{r} \otimes \mathbf{K} \otimes \mathbf{I}_{s}\right)\left(\operatorname{vec} \mathbf{E}_{s, i} \otimes \mathbf{I}_{s^{2}}\right) d \operatorname{vec} \mathbf{B}_{i} \\
d \operatorname{vec} \mathbb{M} & =\sum_{j=1}^{s}\left(\mathbf{I}_{s} \otimes \mathbf{K}^{\top} \otimes \mathbf{I}_{r}\right)\left(\operatorname{vec} \mathbf{E}_{r, j} \otimes \mathbf{I}_{r^{2}}\right) d \operatorname{vec} \mathbf{M}_{j}
\end{aligned}
$$

Substituting (36) and (37) into the expression (33) for $d v e c \mathbf{A}$ gives the final result

$$
\frac{d \operatorname{vec} \mathbf{A}}{d \boldsymbol{\theta}^{\top}}=\underbrace{\mathbf{X}_{1} \sum_{j=1}^{s}\left(\operatorname{vec} \mathbf{E}_{r, j} \otimes \mathbf{I}_{r^{2}}\right) \frac{d \operatorname{vec} \mathbf{M}_{j}}{d \boldsymbol{\theta}^{\top}}}_{\text {perturbations of the } \mathbf{M}_{j}}+\underbrace{\mathbf{X}_{2} \sum_{i=1}^{r}\left(\operatorname{vec} \mathbf{E}_{s, i} \otimes \mathbf{I}_{s^{2}}\right) \frac{d \operatorname{vec} \mathbf{B}_{i}}{d \boldsymbol{\theta}^{\top}}}_{\text {perturbations of the } \mathbf{B}_{i}}
$$

where

$$
\begin{aligned}
& \mathbf{X}_{1}=\left(\mathbb{B}^{\top} \mathbf{K}^{\top} \otimes \mathbf{K}^{\top}\right)\left(\mathbf{I}_{s} \otimes \mathbf{K}^{\top} \otimes \mathbf{I}_{r}\right) \\
& \mathbf{X}_{2}=\left(\mathbf{I}_{s r} \otimes \mathbf{K}^{\top} \mathbf{M} \mathbf{K}\right)\left(\mathbf{I}_{p} \otimes \mathbf{K}^{\top} \otimes \mathbf{I}_{s}\right)
\end{aligned}
$$

Note that $\mathbf{X}_{1}$ and $\mathbf{X}_{2}$ are constant matrices, and need be calculated only once. Although $\mathbf{X}_{1}, \mathbf{X}_{2}$, and the Kronecker products appearing in the summations are large, they are also extremely sparse. The sparse matrix capabilities in MATLAB can take advantage of this fact.

Substituting (38) into (5) gives the sensitivity of an output variable $\boldsymbol{\xi}$ to changes in parameters that perturb the $\mathbf{M}_{j}$, the $\mathbf{B}_{i}$, or both. 


\section{Nonlinear periodic models and delayed density dependence}

We focus here on the sensitivity of the population vector, or functions of the population vector. ${ }^{2}$ These sensitivities have been analyzed in time-invariant nonlinear models (Caswell 2008, 2009a), but not for nonlinear models including periodic environmental variation.

In a periodic nonlinear model, each of the $\mathbf{B}_{i}$ in (2) may depend on density. Especially in seasonal models, the vital rates in the matrix $\mathbf{B}_{i}$ may depend on densities not only at phase $i$, but at previous phases within the cycle as well. For example, the seed production of fruiting plants in the fall may reflect the density experienced by vegetative rosettes in the early spring (Shyu et al. in prep.).

To develop a model including such delayed density dependence, define

$$
\mathbf{n}_{i}(t)=\text { population at season } i \text { in year } t
$$

Starting at season 1 , the dynamics are given by

$$
\begin{aligned}
\mathbf{n}_{1}(t+1) & =\mathbf{B}_{p} \mathbf{n}_{p}(t) \\
\mathbf{n}_{2}(t) & =\mathbf{B}_{1} \mathbf{n}_{1}(t) \\
& \vdots \\
\mathbf{n}_{p}(t) & =\mathbf{B}_{p-1} \mathbf{n}_{p-1}(t)
\end{aligned}
$$

Density-dependence, in a general form, means that the matrices $\mathbf{B}_{i}$ may be functions of densities over one cycle prior to season $i$ :

$$
\begin{aligned}
\mathbf{B}_{1} & =\mathbf{B}_{1}\left[\mathbf{n}_{1}(t), \mathbf{n}_{p}(t-1), \ldots, \mathbf{n}_{2}(t-1)\right] \\
\mathbf{B}_{2} & =\mathbf{B}_{2}\left[\mathbf{n}_{2}(t), \mathbf{n}_{1}(t), \mathbf{n}_{p}(t-1), \ldots, \mathbf{n}_{3}(t-1)\right] \\
& \vdots \\
\mathbf{B}_{p} & =\mathbf{B}_{p}\left[\mathbf{n}_{p}(t), \mathbf{n}_{p-1}(t), \ldots, \mathbf{n}_{1}(t)\right]
\end{aligned}
$$

A fixed point on the interannual time scale is a $p$-cycle on the seasonal scale, satisfying

$$
\begin{aligned}
\hat{\mathbf{n}}_{1} & =\mathbf{B}_{p}\left[\hat{\mathbf{n}}_{1}, \ldots, \hat{\mathbf{n}}_{p}\right] \hat{\mathbf{n}}_{p} \\
\hat{\mathbf{n}}_{2} & =\mathbf{B}_{1}\left[\hat{\mathbf{n}}_{1}, \ldots, \hat{\mathbf{n}}_{p}\right] \hat{\mathbf{n}}_{1} \\
& \vdots \\
\hat{\mathbf{n}}_{p} & =\mathbf{B}_{p-1}\left[\hat{\mathbf{n}}_{1}, \ldots, \hat{\mathbf{n}}_{p}\right] \hat{\mathbf{n}}_{p-1}
\end{aligned}
$$

A $k$-cycle on the interannual time scale is a $k p$-cycle on the seasonal time scale, the points of which we number $\hat{\mathbf{n}}_{1}, \ldots, \hat{\mathbf{n}}_{k p}$. The corresponding sequence of matrices, in which the annual cycle $\mathbf{B}_{1}, \ldots, \mathbf{B}_{p}$ is repeated $k$ times, is defined as $\mathbf{B}_{1}, \ldots, \mathbf{B}_{k p}$. With this notation, (44) still holds, with $k p$ instead of $p$ entries.

Differentiating (44) yields

$$
d \hat{\mathbf{n}}_{i}=\left(d \mathbf{B}_{i-1}\right) \hat{\mathbf{n}}_{i-1}+\mathbf{B}_{i-1}\left(d \hat{\mathbf{n}}_{i-1}\right) \quad i=1, \ldots, p
$$

\footnotetext{
${ }^{2}$ The other main focus of sensitivity analysis for nonlinear models is the invasion exponent in evolutionary applications. The invasion exponent is the dominant eigenvalue of the matrix $\mathbf{A}$ evaluated at equilibrium, or of the periodic product of the matrices over a cycle. Because invasion is a linear problem (Metz et al. 1992), these evolutionary calculations can use (12) directly.
} 
where the subscripts on $\hat{\mathbf{n}}$ and $\mathbf{B}$ are interpreted modulo $p$. Applying the vec operator to (45) yields

$$
d \hat{\mathbf{n}}_{i}=\left(\hat{\mathbf{n}}_{i-1}^{\top} \otimes \mathbf{I}_{s}\right) d \operatorname{vec} \mathbf{B}_{i-1}+\mathbf{B}_{i-1} d \hat{\mathbf{n}}_{i-1} .
$$

The sensitivity analysis of the cycle involves a set of block-structured matrices, the form of which is easily generalized from the special case with $p=3$. Assuming $p=3$ and noting that $\mathbf{B}$ depends on all the $\hat{\mathbf{n}}_{i}$ as well as on the parameter vector $\boldsymbol{\theta}$, the derivative of $\mathbf{B}_{i-1}$ in (46) is

$$
d \operatorname{vec} \mathbf{B}_{i-1}=\frac{\partial \operatorname{vec} \mathbf{B}_{i-1}}{\partial \mathbf{n}_{1}^{\top}} d \hat{\mathbf{n}}_{1}+\frac{\partial \operatorname{vec} \mathbf{B}_{i-1}}{\partial \mathbf{n}_{2}^{\top}} d \hat{\mathbf{n}}_{2}+\frac{\partial \operatorname{vec} \mathbf{B}_{i-1}}{\partial \mathbf{n}_{3}^{\top}} d \hat{\mathbf{n}}_{3}+\frac{\partial \operatorname{vec} \mathbf{B}_{i-1}}{\partial \boldsymbol{\theta}^{\top}} d \boldsymbol{\theta}
$$

For notational convenience, define the matrices

$$
\mathbf{H}_{i}=\left(\hat{\mathbf{n}}_{i}^{\top} \otimes \mathbf{I}_{s}\right)
$$

Substituting (47) into (46) produces the set of equations

$$
\begin{aligned}
d \hat{\mathbf{n}}_{1} & =\mathbf{H}_{3} \frac{\partial \operatorname{vec} \mathbf{B}_{3}}{\partial \boldsymbol{\theta}^{\top}} d \boldsymbol{\theta}+\mathbf{H}_{3} \sum_{j=1}^{p} \frac{\partial \operatorname{vec} \mathbf{B}_{3}}{\partial \mathbf{n}_{j}^{\top}} d \hat{\mathbf{n}}_{j}+\mathbf{B}_{3} d \hat{\mathbf{n}}_{3} \\
d \hat{\mathbf{n}}_{2} & =\mathbf{H}_{1} \frac{\partial \operatorname{vec} \mathbf{B}_{1}}{\partial \boldsymbol{\theta}^{\top}} d \boldsymbol{\theta}+\mathbf{H}_{1} \sum_{j=1}^{p} \frac{\partial \operatorname{vec} \mathbf{B}_{1}}{\partial \mathbf{n}_{j}^{\top}} d \hat{\mathbf{n}}_{j}+\mathbf{B}_{1} d \hat{\mathbf{n}}_{1} \\
d \hat{\mathbf{n}}_{1} & =\mathbf{H}_{2} \frac{\partial \operatorname{vec} \mathbf{B}_{2}}{\partial \boldsymbol{\theta}^{\top}} d \boldsymbol{\theta}+\mathbf{H}_{2} \sum_{j=1}^{p} \frac{\partial \operatorname{vec} \mathbf{B}_{2}}{\partial \mathbf{n}_{j}^{\top}} d \hat{\mathbf{n}}_{j}+\mathbf{B}_{2} d \hat{\mathbf{n}}_{2}
\end{aligned}
$$

This set of equations can be reduced to a single equation by collecting all the points on the $k p$-cycle into a single vector. Write an array (of dimension $s p \times k$ )

$$
\mathcal{N}=\begin{array}{c|ccc} 
& \text { yr. 1 } & & \text { yr. } k \\
\hline \text { season 1 } & \hat{\mathbf{n}}_{1} & \cdots & \hat{\mathbf{n}}_{1} \\
\vdots & \vdots & & \vdots \\
\text { season } p & \hat{\mathbf{n}}_{p} & \cdots & \hat{\mathbf{n}}_{p} \\
\hline
\end{array}
$$

Then write the vector (of dimension $s p k \times 1$ )

$$
\mathbb{N}=\operatorname{vec} \mathcal{N}
$$

In terms of this vector, the set of equations (49) can be rewritten

$$
\frac{d \mathbb{N}}{d \boldsymbol{\theta}^{\top}}=\left[\mathbf{I}_{s k p}-\mathbb{B}-\mathbb{H} \mathbb{C}\right]^{-1} \mathbb{H} \mathbb{D} .
$$

where $\mathbb{H}$ and $\mathbb{B}$ are the block-circulant matrices

$$
\begin{aligned}
\mathbb{H} & =\left(\begin{array}{ccc}
0 & 0 & \mathbf{H}_{3} \\
\mathbf{H}_{1} & 0 & 0 \\
0 & \mathbf{H}_{2} & 0
\end{array}\right) \\
\mathbb{B} & =\left(\begin{array}{ccc}
0 & 0 & \mathbf{B}_{3} \\
\mathbf{B}_{1} & 0 & 0 \\
0 & \mathbf{B}_{2} & 0
\end{array}\right),
\end{aligned}
$$


and $\mathbb{C}$ and $\mathbb{D}$ are the block matrices

$$
\begin{aligned}
& \mathbb{C}=\left(\begin{array}{ccc}
\frac{\partial \operatorname{vec} \mathbf{B}_{1}}{\partial \mathbf{n}_{1}^{\top}} & \cdots & \frac{\partial \operatorname{vec} \mathbf{B}_{1}}{\partial \mathbf{n}_{3}^{\top}} \\
\vdots & \ddots & \vdots \\
\frac{\partial \operatorname{vec} \mathbf{B}_{3}}{\partial \mathbf{n}_{1}^{\top}} & \cdots & \frac{\partial \operatorname{vec} \mathbf{B}_{3}}{\partial \mathbf{n}_{3}^{\top}}
\end{array}\right) \\
& \mathbb{D}=\left(\begin{array}{c}
\frac{\partial \operatorname{vec} \mathbf{B}_{1}}{\partial \boldsymbol{\theta}^{\top}} \\
\frac{\partial \operatorname{vec} \mathbf{B}_{2}}{\partial \boldsymbol{\theta}^{\top}} \\
\frac{\partial \operatorname{vec} \mathbf{B}_{3}}{\partial \boldsymbol{\theta}^{\top}}
\end{array}\right) .
\end{aligned}
$$

All the derivatives are evaluated at $\hat{\mathbf{n}}_{1}, \ldots, \hat{\mathbf{n}}_{3}$. This derivation follows that in Caswell (2008), but with the addition of delayed density dependence.

\subsection{Averages}

The vector $d \mathbb{N} / d \boldsymbol{\theta}^{\top}$ contains the sensitivities of all $s$ stages at each of $p$ seasons with the year (for a fixed point on the annual time scale) or at each of $k p$ seasons for a $k$-cycle on the annual time scale. That may be too much information; it may be desirable to calculate the sensitivity of averages, or other linear combinations, taken in various ways.

To write these averages, let $\mathbf{b}_{m}$ be a $m \times 1$ vector of weights. For a simple average of $m$ quantities, each entry of $\mathbf{b}_{m}$ is $1 / m$; for a weighted average, the entries of $\mathbf{b}_{m}$ would be non-negative numbers summing to 1 . More generally, $\mathbf{b}$ may contain arbitrary weights, such as biomass, metabolic rate, economic value, etc. (Caswell 2008). To calculate averages from $\mathbb{N}$, we apply these vectors to $\mathcal{N}$ and then apply the vec operator.

Given a fixed point on the annual time scale, averages can be calculated over stages (using a vector $\mathbf{b}_{s}$ ), over seasons (using a vector $\mathbf{b}_{p}$ ), or both. The $p \times 1$ vector of averages over stages is

$$
\begin{aligned}
\text { avg. over stages } & =\operatorname{vec}\left(\mathbf{b}_{s}^{\top} \mathcal{N}\right) \\
& =\left(\mathbf{I}_{p} \otimes \mathbf{b}_{s}^{\top}\right) \mathbb{N}
\end{aligned}
$$

The $s \times 1$ vector of averages over seasons is

$$
\text { avg. over seasons }=\left(\mathbf{b}_{p}^{\top} \otimes \mathbf{I}_{s}\right) \mathbb{N}
$$

The average over both seasons and stages (a scalar) is

$$
\text { avg. over stages and seasons }=\left(\mathbf{b}_{p}^{\top} \otimes \mathbf{b}_{s}^{\top}\right) \mathbb{N}
$$

Because the average is a linear operator, the sensitivities of these averages are obtained by applying the same weights to $d \mathbb{N} / d \boldsymbol{\theta}^{\top}$ in (52):

$$
\begin{aligned}
\text { sensitivity of avg. over stages } & =\left(\mathbf{I}_{p} \otimes \mathbf{b}_{s}^{\top}\right) \frac{d \mathbb{N}}{d \boldsymbol{\theta}^{\top}} \\
\text { sensitivity of avg. over seasons } & =\left(\mathbf{b}_{p}^{\top} \otimes \mathbf{I}_{s}\right) \frac{d \mathbb{N}}{d \boldsymbol{\theta}^{\top}} \\
\text { sensitivity of avg. over both } & =\left(\mathbf{b}_{p}^{\top} \otimes \mathbf{b}_{s}^{\top}\right) \mathbb{N}
\end{aligned}
$$


Table 2: Calculation of averages of attractors of nonlinear periodic matrix population models. The upper half of the table shows averages over stages and over seasons when the dynamics are a fixed point on the inter-annual time scale, and thus a $p$-cycle on the seasonal time scale. The lower half of the table shows averages over all combinations of stages, seasons, and years, when the dynamics are a $k$-cycle on the inter-annual time scale, and thus a $k p$-cycle on the seasonal time scale.

\begin{tabular}{l|c|c|c} 
Average over: & Formula & Vectors & Dimension \\
\hline \hline \multirow{2}{*}{ stages } & $\left(\mathbf{I}_{p} \otimes \mathbf{b}_{s}^{\top}\right) \mathbb{N}$ & 1 & $p \times 1$ \\
seasons & $\left(\mathbf{b}_{p}^{\top} \otimes \mathbf{I}_{s}\right) \mathbb{N}$ & 1 & $s \times 1$ \\
seasons and stages & $\left(\mathbf{b}_{p}^{\top} \otimes \mathbf{b}_{s}\right) \mathbb{N}$ & 1 & $1 \times 1$ \\
\hline stages & $\left(\mathbf{I}_{k} \otimes \mathbf{I}_{p} \otimes \mathbf{b}_{s}^{\top}\right) \mathbb{N}$ & 1 & $k p \times 1$ \\
seasons & $\left(\mathbf{I}_{k} \otimes \mathbf{b}_{p}^{\top} \otimes \mathbf{I}_{s}\right) \mathbb{N}$ & $k$ & $s \times 1$ \\
years & $\left(\mathbf{b}_{k} \otimes \mathbf{I}_{p} \otimes \mathbf{I}_{s}\right) \mathbb{N}$ & $p$ & $s \times 1$ \\
seasons and years & $\left(\mathbf{b}_{k}^{\top} \otimes \mathbf{b}_{p}^{\top} \otimes \mathbf{I}_{s}\right) \mathbb{N}$ & 1 & $s \times 1$ \\
seasons and stages & $\left(\mathbf{I}_{k} \otimes \mathbf{b}_{p}^{\top} \otimes \mathbf{b}_{s}^{\top}\right) \mathbb{N}$ & 1 & $k \times 1$ \\
years and stages & $\left(\mathbf{b}_{k}^{\top} \otimes \mathbf{I}_{p} \otimes \mathbf{b}_{s}^{\top}\right) \mathbb{N}$ & 1 & $p \times 1$ \\
stages, seasons, years & $\left(\mathbf{b}_{k}^{\top} \otimes \mathbf{b}_{p}^{\top} \otimes \mathbf{b}_{s}^{\top}\right) \mathbb{N}$ & 1 & $1 \times 1$
\end{tabular}

When the dynamics produce a $k$-cycle on the annual time scale, averages can be calculated over any desired combination of stages, seasons, and years. Table 2 gives the resulting expressions for the averages. As in the case of equations (61) and (62), the sensitivities of these averages to parameters are obtained by applying the same weights to $d \mathbb{N} / d \boldsymbol{\theta}^{\top}$.

\section{$4.2 \quad$ A nonlinear example}

As an example of the calculations for nonlinear systems, imagine an organism with two stages: immature juveniles and reproducing adults. Suppose that the year contains two seasons: a benign, reproduction-heavy Season 1 and a harsh, mortality-heavy Season 2. The life cycle graph is shown in Figure 2. Adults in Season 1 all survive to Season 2 and give birth to new juveniles with percapita fertility $f$, which depends on adult density in Season 1 according to $f\left[\mathbf{n}_{1}\right]=a e^{-b n_{2}}$, where $a$ and $b$ are the maximum fertility and the strength of density-dependence, respectively, and $n_{2}$ is the adult density in Season 1. In the harsher Season 2, juveniles and adults survive with probabilities $s_{j}$ and $s_{a}$. A juvenile that survives to Season 1 matures into an adult.

This life cycle produces seasonal transition matrices $\mathbf{B}_{1}$ and $\mathbf{B}_{2}$ :

$$
\begin{aligned}
\mathbf{B}_{1}\left[\mathbf{n}_{1}\right] & =\left(\begin{array}{cc}
0 & f\left[\mathbf{n}_{1}\right] \\
0 & 1
\end{array}\right) \\
\mathbf{B}_{2} & =\left(\begin{array}{cc}
0 & 0 \\
s_{j} & s_{a}
\end{array}\right)
\end{aligned}
$$

and the nonlinear periodic model

$$
\mathbf{n}_{1}(t+1)=\mathbf{B}_{2} \mathbf{n}_{2}(t)
$$




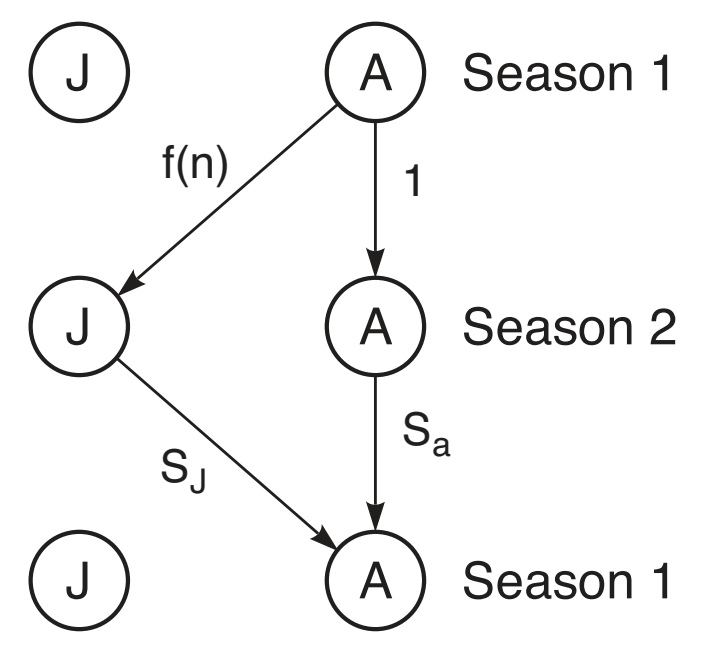

Figure 2: A periodic life cycle graph for a simple two-stage, two-season nonlinear model. $J$ and $A$ denote juveniles and adults, respectively.

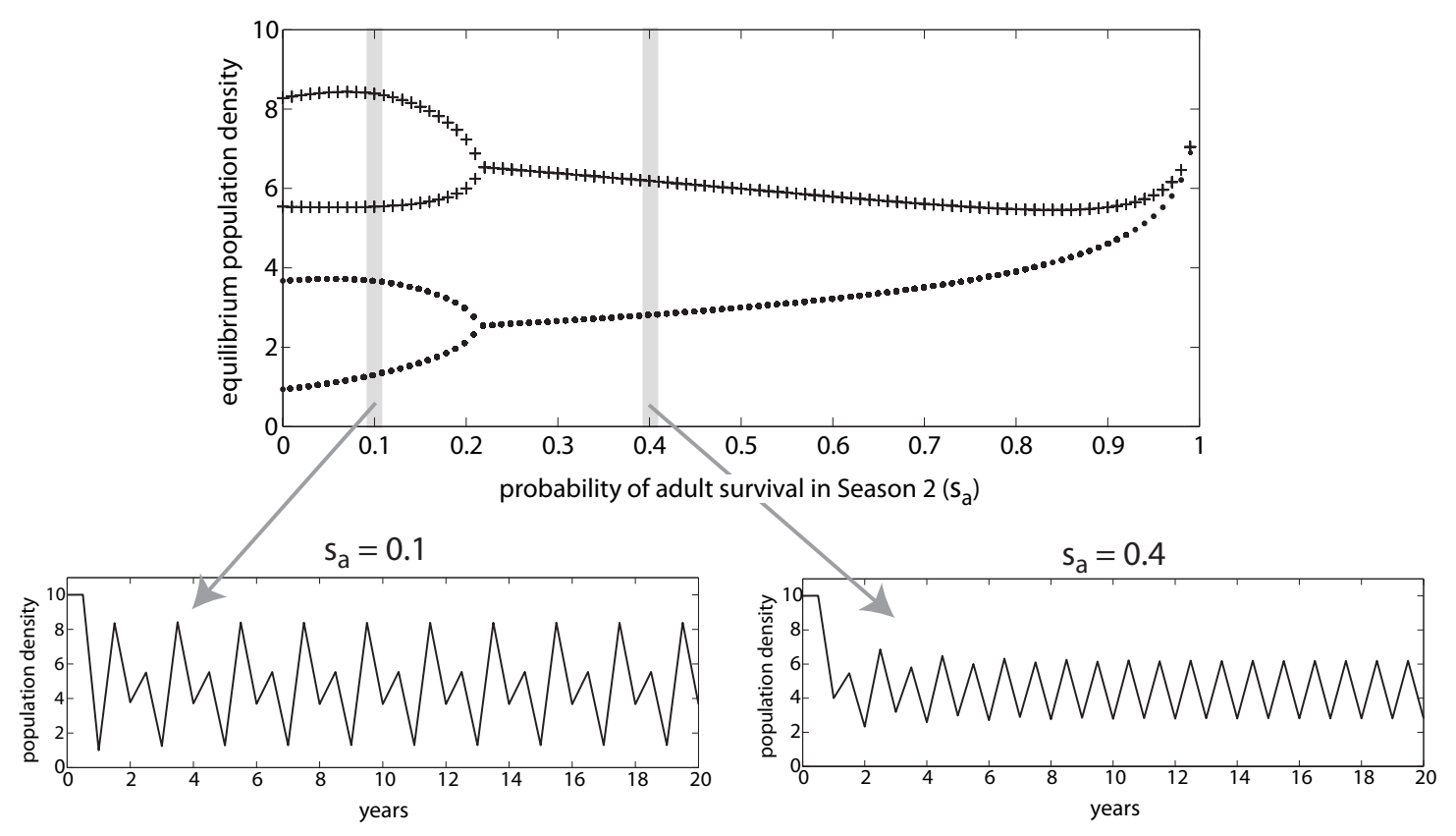

Figure 3: A bifurcation diagram on the seasonal time scale for the two-season, two-stage model of Figure 2. Total densities are plotted for Season $1(\bullet)$ and $2(+)$. Parameters: $a=20, b=1$, $s_{j}=0.5 ; s_{a}$ varied from 0 to 1 .

$$
\mathbf{n}_{2}(t)=\mathbf{B}_{1}\left[\mathbf{n}_{1}(t)\right] \mathbf{n}_{1}(t)
$$

Figure 3 is a bifurcation diagram for the system (66) in response to changes in adult survival $s_{a}$. When adults are long-lived $\left(s_{a} \gtrsim 0.22\right)$ there is a 2-cycle on the seasonal scale, corresponding to a 
fixed point on the annual time scale, satisfying

$$
\begin{aligned}
& \hat{\mathbf{n}}_{1}=\mathbf{B}_{2} \hat{\mathbf{n}}_{2} \\
& \hat{\mathbf{n}}_{2}=\mathbf{B}_{1}\left[\hat{\mathbf{n}}_{1}\right] \hat{\mathbf{n}}_{1}
\end{aligned}
$$

At $s_{a} \approx 0.22$ this 2 -cycle bifurcates to a 4 -cycle on the seasonal time scale, corresponding to a 2-cycle on the annual scale.

To derive the block matrices $\mathbb{C}$ and $\mathbb{D}$ in equations (55) and (56), we define the parameter vector as

$$
\boldsymbol{\theta}=\left(\begin{array}{llll}
s_{j} & s_{a} & a & b
\end{array}\right)^{\top} .
$$

The derivative matrices are

$$
\begin{aligned}
\frac{d \operatorname{vec} \mathbf{B}_{1}}{d \boldsymbol{\theta}^{\top}} & =\left(\begin{array}{cccc}
0 & 0 & 0 & 0 \\
0 & 0 & 0 & 0 \\
0 & 0 & e^{-b \hat{n}_{2}} & -a \hat{n}_{2} e^{-b \hat{n}_{2}} \\
0 & 0 & 0 & 0
\end{array}\right) \\
\frac{d \operatorname{vec} \mathbf{B}_{2}}{d \boldsymbol{\theta}^{\top}} & =\left(\begin{array}{cccc}
0 & 0 & 0 & 0 \\
1 & 0 & 0 & 0 \\
0 & 0 & 0 & 0 \\
0 & 1 & 0 & 0
\end{array}\right) \\
\frac{d \operatorname{vec} \mathbf{B}_{1}}{d \mathbf{n}^{\top}} & =\left(\begin{array}{cc}
0 & 0 \\
0 & 0 \\
0 & -a b e^{-b \hat{n}_{2}} \\
0 & 0
\end{array}\right) \\
\frac{d \operatorname{vec} \mathbf{B}_{2}}{d \mathbf{n}^{\top}} & =\mathbf{0} \text { (dimension } 4 \times 2)
\end{aligned}
$$

We calculate the sensitivities of the equilibrium population at each phase of the cycle using equation (52) with $s_{a}=0.4$ (a 2-cycle on the seasonal time scale; see Figure 3) and with $s_{a}=0.1$ (a 4-cycle on the seasonal time scale). The results, and the sensitivities of several averages, are shown in Figure 4.

At the seasonal 2-cycle (annual fixed point), increases in $s_{j}$ or $s_{a}$ increase density in Season 1 and reduce density in Season 2, and have little effect on the density averaged over seasons. The maximum fertility level $a$ has little effect at either season, and the density-dependent parameter $b$ has large negative effects throughout.

At the 4-point seasonal cycle (2-cycle on the annual time scale), the patterns are more complicated. We describe them in terms of the $k p=4$ seasons in the cycle. The maximum fertility $a$ has little effect at any point. The survival probabilities $s_{j}$ and $s_{a}$ have effects that are opposite in sign: an increase in $s_{j}$ increases the density in seasons 1 and 4 , and reduces it in seasons 2 and 3 . An increase in $s_{a}$ has the opposite effect. Averaged over years, both $s_{a}$ and $s_{j}$ increase density in season 1 and reduce it in season 2, thus increasing the amplitude of the oscillation. Averaged over seasons, $s_{a}$ and $s_{j}$ have opposite effects. When averaged over stages, seasons, and years, the effects of $s_{a}$ cancel each other out, and only $s_{j}$ and $b$ have appreciable effects.

Even in this simple example, it is clear that parameter changes can have effects that differ among seasons and years. A set of MATLAB scripts to carry out these calculations is provided in an Online Supplement. 

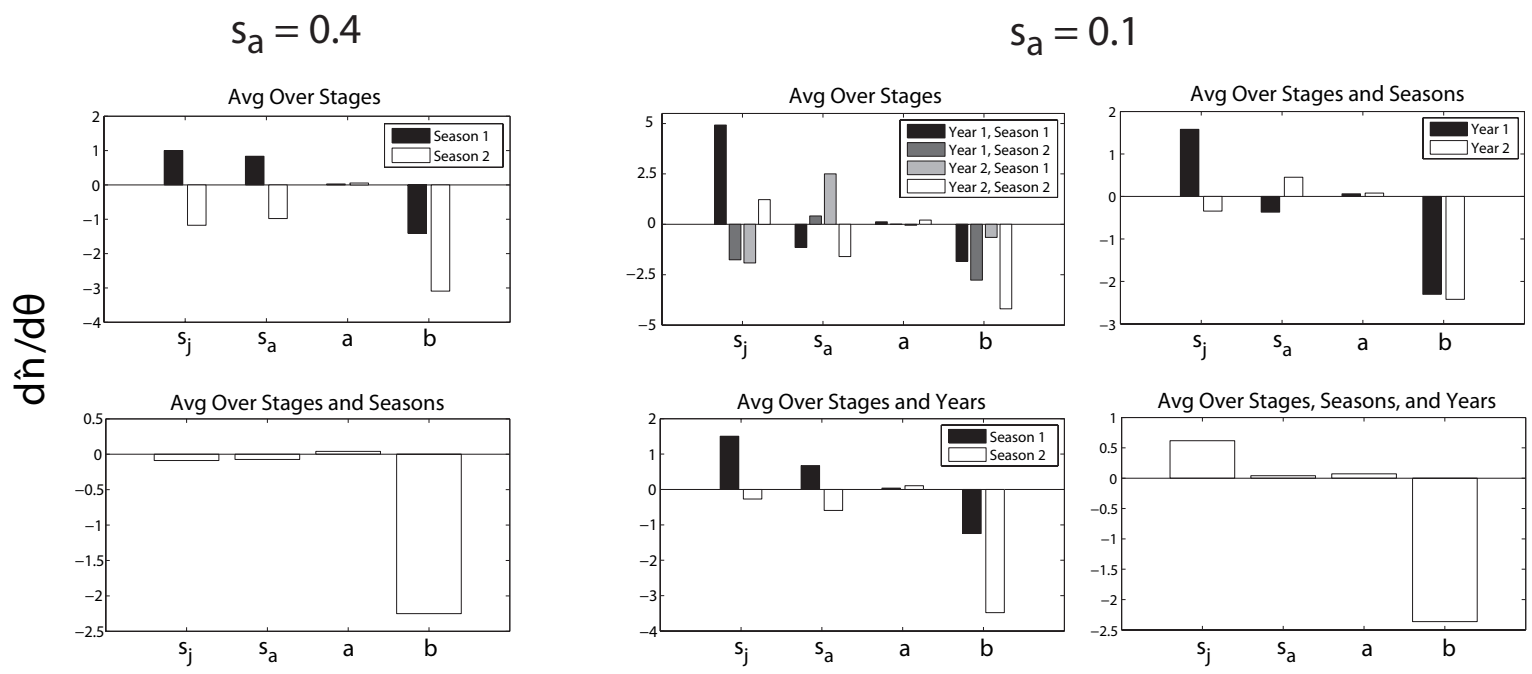

Figure 4: Sensitivities of equilibrium total population size in Seasons 1 and 2, as well as the annual population average, to the demographic parameters $s_{j}, s_{a}, a$, and $b$. Left: sensitivities when $s_{a}$ $=0.4$ (seasonal 2-cycle, annual equilbrium). Right: sensitivities when $s_{j}=0.1$ (seasonal 4-cycle, annual 2-cycle).

\section{An application: LTRE Analysis}

LTRE (Life Table Response Experiment) analysis provides a first-order decomposition of effects on a dependent variable, such as $\lambda$, into contributions from differences in the parameters (Caswell 1989, 2001 Chapter 10). In the case of a periodic model, the effects on the dependent variable result from differences in each parameter at each phase in the cycle (Smith et al. 2005). The perturbation analysis presented here makes it possible to decompose differences in the dependent variable into contributions from the differences in each parameter.

Suppose that $\boldsymbol{\xi}$ is a $m \times 1$ dependent variable (scalar or vector-valued), a function of a vector of parameters $\boldsymbol{\theta}$ that takes on values $\boldsymbol{\theta}_{1}, \ldots, \boldsymbol{\theta}_{p}$ over the cycle. Use superscripts to denote two treatments, which produce results $\boldsymbol{\xi}^{(1)}$ and $\boldsymbol{\xi}^{(2)}$ :

$$
\begin{aligned}
& \boldsymbol{\theta}_{1}^{(1)}, \ldots, \boldsymbol{\theta}_{p}^{(1)} \rightarrow \boldsymbol{\xi}^{(1)} \\
& \boldsymbol{\theta}_{1}^{(2)}, \ldots, \boldsymbol{\theta}_{p}^{(2)} \rightarrow \boldsymbol{\xi}^{(2)}
\end{aligned}
$$

To first order, the treatment effect on $\boldsymbol{\xi}$ is

$$
\boldsymbol{\xi}^{(2)}-\boldsymbol{\xi}^{(1)} \approx \sum_{i=1}^{p} \frac{d \boldsymbol{\xi}}{d \boldsymbol{\theta}_{i}^{\top}}\left(\boldsymbol{\theta}_{i}^{(2)}-\boldsymbol{\theta}_{i}^{(1)}\right)
$$

The $i$ th term in the summation in (76) is the total contribution, over all of the parameters in

$\boldsymbol{\theta}$, of parameter differences in phase $i$ of the cycle. This can be expanded to form a matrix of contributions, $\mathbf{R}_{i}$, whose $(k, j)$ entry is the contribution of the $j$ th entry in the parameter vector $\boldsymbol{\theta}$, at phase $i$, to the effect on the $k$ th entry of the dependent variable vector $\boldsymbol{\xi}$ :

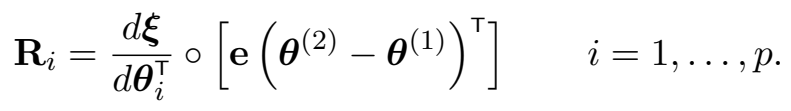


Table 3: The major results of this paper; for each calculation, the equation number for the sensitivity is given.

\begin{tabular}{lc}
\multicolumn{1}{c}{ Model } & Sensitivity \\
\hline Linear periodic model & $(12)$ \\
Harvest model & $(19)$ \\
Seasonal harvest model & $(22)$ \\
Vec-permutation model & $(38)$ \\
Nonlinear model & $(52)$ \\
Averages over cycles & Table 2 \\
LTRE contributions & $(77)$
\end{tabular}

Here, o denotes the Hadamard product and $\mathbf{e}$ is a $m \times 1$ vector of ones. If $\boldsymbol{\xi}$ is a scalar, then $\mathbf{R}_{k}$ is a row vector of contributions. The derivative is evaluated at the average of $\boldsymbol{\theta}^{(1)}$ and $\boldsymbol{\theta}^{(2)}$.

The contribution matrix (77) requires $d \boldsymbol{\xi} / d \boldsymbol{\theta}_{i}^{\top}$, that is, the derivative of $\boldsymbol{\xi}$ to the parameter at phase $i$ of the cycle. In the linear model (2), this is given by the $i$ th term in the summation in (12). In the case of the nonlinear model (42), the derivative is obtained from equation (52) by setting all blocks of $\mathbb{D}$, except those corresponding to phase $i$, to zero.

\section{Discussion}

The distinguishing feature of periodic models is that the dynamics over a projection interval are given by a periodic product of matrices. The periodic product may reflect the existence of multiple timescales (e.g., seasonal and annual), or the operation of multiple processes (e.g., demography and harvest), or express conditional probabilities, or arise from classifying individuals by multiple criteria. The sensitivity analysis of periodic models must account for the chain of causation shown in Figure 1. The underlying parameters affect the vital rates at each phase of the cycle, but the output variable $\boldsymbol{\xi}$ is calculated from the entire periodic product. Differentiating $\boldsymbol{\xi}$ with respect to $\boldsymbol{\theta}$ requires tracing all these chains of influence. Matrix calculus makes this easy to do, starting with a simple chain rule expression [see equation (5)] and then using the periodic form to calculate $d \operatorname{vec} \mathbf{A} / d \boldsymbol{\theta}^{\top}$, as in equation (11) for linear models, (22) for seasonal harvest models, and (38) for vec-permutation models. Sensitivity analysis of nonlinear models is equally direct, with equation (52) combining all the points on the seasonal or interannual cycles into a single vector.

An overview of the results we have obtained here is given in Table 3.

Analytical results such as we present here are always preferable, both theoretically and practically, to numerical approximations. It is even better to have both. This is particularly true when the analytical results are easily computable, and is especially true when applied to differentiation, which is a notoriously ill-behaved problem. The naive numerical approach, based on small perturbations of each parameter in turn, is "almost guaranteed to produce inaccurate results" (Press et al. 1992, p. 185). It is subject to truncation error (caused by making the perturbation too large) and roundoff error (caused by making the perturbation too small). In some applications these errors will be unimportant, but in others they can be crucial (c.f. Hunter and Caswell (2009) for an example in mark-recapture analysis).

In management applications, the timing of interventions can be as much a part of the strategy as the choice of stages to target. The sensitivity formulae derived here explicitly account for 
perturbations at any point in the periodic product. This permits investigation of season-specific management strategies (e.g., Shyu et al. in prep.). Combining seasonality and the vec-permutation model would permit the analysis of strategies that are both season and location-specific. Brooks and Lebreton (2001), for example, carried out a detailed analysis of control strategies for a gull metapopulation. They developed optimal stage- and site-specific harvest strategies using sensitivity analysis of the population growth rate $\lambda$. It would be interesting to develop those calculations using the periodic sensitivity analysis of the corresponding vec-permutation model.

\section{Acknowledgments}

This research was supported by NSF Grant DEB-0816514, by a Research Award from the Alexander von Humboldt Foundation, and by WHOI Academic Programs Funds. HC is grateful to the Max Planck Institute for Demographic Research for hospitality while these ideas were developed, and also thanks Samtrot. We thank T. Knight and E. Pardini for their collaboration on application of some of these results to invasive species control, and an anonymous reviewer for helpful comments.

\section{$7 \quad$ Literature cited}

Abadir, K. M. and J. R. Magnus. 2005. Matrix algebra. Cambridge University Press, New York, New York, USA.

Awkerman J. A., K. P. Huyvaert, J. Mangel, J. A. Shigueto, and D. J. Anderson. 2006. Incidental and intentional catch threatens Galàpagos waved albatross. Biological Conservation 133:483489.

Blomberg, S. P. and R. Shine. 2001. Modelling life history strategies with capture-recapture data: Evolutionary demography of the water skink Eulamprus tympanum. Austral Ecology 26:349359.

Brooks, E.N. and J.-D. Lebreton. 2001. Optimizing removals to control a metapopulation: application to the yellow legged herring gull (Larus cachinnans). Ecological Modelling 136:269-284.

Caswell, H. 2001. Matrix population models: construction, analysis, and interpretation. Second edition. Sinauer Associates, Sunderland, Massachusetts, USA.

Caswell, H. 2006. Applications of Markov chains in demography. pp. 319-334 in A.N. Langville and W.J. Stewart (eds.)MAM2006: Markov Anniversary Meeting. Boson Books, Raleigh, North Carolina, USA.

Caswell, H. 2007. Sensitivity analysis of transient population dynamics. Ecology Letters 10:1-15.

Caswell, H. 2008. Perturbation analysis of nonlinear matrix population models. Demographic Research. 18:59-116.

Caswell, H. 2009a. Sensitivity and elasticity of density-dependent population models. Journal of Difference Equations and Applications 15:349-369.

Caswell, H. 2009b. Stage, age, and individual stochasticity in demography. Oikos 118:1763-1782.

Caswell, H. 2010. Reproductive value, the stable stage distribution, and the sensitivity of the population growth rate to changes in vital rates. Demographic Research 23:531-548.

Caswell, H. 2011a. Beyond $R_{0}$ : Demographic models for variability of lifetime reproductive output. PLoS ONE 6: e20809. doi:10.1371/journal.pone.0020809

Caswell, H. 2011b. Matrix models and sensitivity analysis of populations classified by age and stage: a vec-permutation matrix approach. Theoretical Ecology 1-15.

Caswell, H. 2011c. Sensitivity analysis of discrete Markov chains via matrix calculus. Linear Algebra and its Applications (in press). 
Caswell, H. and T. N. Kaye. 2001. Stochastic demography and conservation of an endangered perennial plant (Lomatium bradshawii) in a dynamic fire regime. Advances in Ecological Research 32:1-51.

Caswell, H. and M. C. Trevisan. 1994. Sensitivity analysis of periodic matrix models. Ecology $75: 1299-1303$.

Cushing, J.M. 2006. A juvenile-adult model with periodic vital rates. Journal of Mathematical Biology 53:520-539.

Darwin, J. H. and R. M. Williams. 1964. The effect of time of hunting on the size of a rabbit population. New Zealand Journal of Science 7:341-352.

Davis, A. S., P. M. Dixon, and M. Liebman. 2003. Cropping system effects on giant foxtail (Setaria faberi) demography: II. Retrospective perturbation analysis. Weed Science 51:930-939.

Davis, A. S., P. M. Dixon, and M. Liebman. 2004. Using matrix models to determine cropping system effects on annual weed demography. Ecological Applications 14:655-668.

Gervais, J. A., C. M. Hunter, and R. G. Anthony. 2006. Interactive effects of prey and p,p '-DDE on burrowing owl population dynamics. Ecological Applications 16:666-677.

Goldberg EE, Lynch HJ, Neubert MG, Fagan WF (2010) Effects of branching spatial structure and life history on the asymptotic growth rate of a population. Theoretical Ecology 3:137-152.

Golubov, J., M. D. Mandujano, M. Franco, C. Montana, L. E. Eguiarte, and J. Lopez-Portillo. 1999. Demography of the invasive woody perennial Prosopis glandulosa (honey mesquite). Journal of Ecology 87:955-962.

Griffith, A. B., and M. E. Loik. 2010. Effects of climate and snow depth on Bromus tectorum population dynamics at high elevation. Oecologia 164:821-832.

Gross, K., J. R. Lockwood, C. C. Frost, and M. F. Morris. 1998. Modeling controlled burning and trampling reduction for conservation of Hudsonia montana. Conservation Biology 12:1291-1301.

Hauser, C. E., E. G. Cooch, and J. D. Lebreton. 2006. Control of structured populations by harvest. Ecological Modelling 196:462-470.

Henderson, H. V. and S. R. Searle. 1981. The vec-permutation matrix, the vec operator and Kronecker products: a review. Linear and Multilinear Algebra 9:271-288.

Henson, S.M. and J.M. Cushing. 1997. The effect of periodic habitat fluctuations on a nonlinear insect population model. Journal of Mathematical Biology 36:201-226.

Hill, M. F., J. D. Witman, and H. Caswell. 2004. Markov chain analysis of succession in a rocky subtidal community. The American Naturalist 164:46-61.

Hoffmann, W. A. 1999. Fire and population dynamics of woody plants in a neotropical savanna: matrix model projections. Ecology 80:1354-1369.

Horvitz, C.C. and S. Tuljapurkar. 2008. Stage dynamics, period survival, and mortality plateaus. American Naturalist 172:203-215.

Hunter, C. M. and H. Caswell. 2005a. Selective harvest of sooty shearwater chicks: effects on population dynamics and sustainability. Journal of Animal Ecology 74:589-600.

Hunter, C.M. and H. Caswell. 2005b. The use of the vec-permutation matrix in spatial matrix population models. Ecological Modelling 188:15-21.

Hunter, C.M. and H. Caswell. 2009. Rank and redundancy of multistate mark-recapture models for seabird populations with unobservable states. Modeling Demographic Processes in Marked Populations. D. Thomson, E.G. Cooch, and M.J. Conroy, eds. Ecological and Environmental Statistics 3:797-825.

Hyatt, L. A. and S. Araki. 2006. Comparative population dynamics of an invading species in its native and novel ranges. Biological Invasions 8:261-275.

Jenouvrier, S., H. Caswell, C. Barbraud, and H. Weimerskirch. 2010. Mating behavior, population growth, and the operational sex ratio: a periodic two-sex model approach. The American 
Naturalist 175:739-752.

Klepac, P. and H. Caswell. 2010. The stage-structured epidemic: linking disease and demography with a multi-state matrix approach model. Theoretical Ecology 4:301-319.

Le Corff, J. and C. C. Horvitz. 2005. Population growth versus population spread of an antdispersed neotropical herb with a mixed reproductive strategy. Ecological Modelling 188:41-51.

Lebreton, J.-D. 1996. Demographic models for subdivided populations: the renewal equation approach. Theoretical Population Biology 49:291-313.

Lebreton, J.-D. and P. Isenmann. 1976. Dynamique de la population carmaguaise de Mouette rieuse: un modéle mathématique. La Terre et La Vie (Revue d'Écologie) 30: 529-549.

Lebreton, J.-D. and R. Pradel. 2002. Multistate recapture models: modelling incomplete individual histories. Journal of Applied Statistics 29:353-369.

Lesnoff, M. 1999. Dynamics of a sheep population in a Sahelian area (Ndiagne district in Senegal): a periodic matrix model. Agricultural Systems. 61:207-221.

Lesnoff, M., R. Lancelot, E. Tillard, and I. R. Dohoo. 2000. A steady-state approach of benefit-cost analysis with a periodic Leslie-matrix model: Presentation and application to the evaluation of a sheep-diseases preventive scheme in Kolda, Senegal. Preventive Veterinary Medicine: 46:113128.

Lesnoff, M., P. Ezanno, and H. Caswell. 2003. Sensitivity analysis in periodic matrix models: a postscript to Caswell and Trevisan. Mathematical and Computer Modelling 37: 945-948.

Lima, M., N. C. Stenseth, H. Leirs, and F. M. Jaksic. 2003. Population dynamics of small mammals in semi-arid regions: a comparative study of demographic variability in two rodent species. Proceedings of the Royal Society of London B 270:1997-2007.

Lima, M., N. C. Stenseth, N. G. Yoccoz, and F. M. Jaksic. 2001. Demography and population dynamics of the mouse opossum (Thylamys elegans) in semi-arid Chile: seasonality, feedback structure and climate. Proceedings of the Royal Society B 268:2053-2064.

MacArthur, R. H. 1968. Selection for life tables in periodic environments. The American Naturalist 102:381-383.

Magnus, J.R. and H. Neudecker. 1979. The commutation matrix: some properties and applications. Annals of Statistics 7:381-394.

Magnus, J. R. and H. Neudecker. 1985. Matrix differential calculus with applications to simple, Hadamard, and Kronecker products. Journal of Mathematical Psychology 29:474-492.

Mandujano, M. C., C. Montana, M. Franco, J. Golubov, and A. Flores-Martinez. 2001. Integration of demographic annual variability in a clonal desert cactus. Ecology 82:344-359.

Mertens S. K., F. van den Bosch, and J. A. P. Heesterbeek. 2002. Weed populations and crop rotations: Exploring dynamics of a structured periodic system. Ecological Applications. 12:11251141.

Metz, J.A.J., R.M. Nisbet, and S.A.H. Geritz. 1992. How should we define 'fitness' for general ecological scenarios? Trends in Ecology and Evolution 7:198-202.

Mondragon, D., R. Duran, I. Ramirez, and T. Valverde. 2004. Temporal variation in the demography of the clonal epiphyte Tillandsia brachycaulos (Bromeliaceae) in the Yucatan Peninsula, Mexico. Journal of Tropical Ecology 20:189-200.

Ozgul A, Oli MK, Armitage KB, Blumstein DT, van Vuren DH (2009) Influence of local demography on asymptotic and transient dynamics of a yellow-bellied marmot metapopulation. American Naturalist 173:517-530.

Pagel, J., K. Fritzsch, R. Biedermann, and B. Schroeder. 2008. Annual plants under cyclic disturbance regime: better understanding through model aggregation. Ecological Applications 18:2000-2015. 
Pascarella, J. B., and C. C. Horvitz. 1998. Hurricane disturbance and the population dynamics of a tropical understory shrub: mega-matrix elasticity analysis. Ecology 79:547-563.

Pico, F. X., H. de Kroon, and J. Retana. 2002. An extended flowering and fruiting season has few demographic effects in a Mediterranean perennial herb. Ecology 83:1991-2004.

Press, W.H., S.A. Teukolsky, W.T. Vetterling, and B.P. Flannery. 1992. Numerical recipes in C: the art of scientific computing. Second Edition. Cambridge University Press, Cambridge, United Kingdom.

Prevey, J. S., M. J. Germino, and N. J. Huntly. 2010. Loss of foundation species increases population growth of exotic forbs in sagebrush steppe. Ecological Applications 20:1890-1902.

Ramula, S. 2008. Responses to the timing of damage in an annual herb: Fitness components versus population performance. Basic and Applied Ecology 9:233-242.

Ramula, S. and Y. M. Buckley. 2010. Management recommendations for short-lived weeds depend on model structure and explicit characterization of density dependence. Methods in Ecology and Evolution 1: 158-167.

Ripley, B. J., J. Holtz and M. A. Simovich. 2004. Cyst bank life-history model for a fairy shrimp from ephemeral ponds. Freshwater Biology 49:221-231.

Rydgren, K., H. de Kroon, R. H. Okland, and J. van Groenendael. 2001. Effects of fine-scale disturbances on the demography and population dynamics of the clonal moss Hylocomium splendens. Journal of Ecology 89:395-405.

Sarukhan, J. and M. Gadgil. 1974. Studies on plant demography: Ranunculus repens L., R. bulbosus L., and $R$. acris L. III. A mathematical model incorporating multiple modes of reproduction. Journal of Ecology 62:921-936.

Schleuning, M., V. Huaman, and D. Matthies. 2008. Flooding and canopy dynamics shape the demography of a clonal Amazon understorey herb. Journal of Ecology. 96: 1045-1055.

Shyu, E., E.A. Pardini, T.M. Knight, and H. Caswell. (in prep.) A seasonal, density-dependent, stage-structured harvest model for the management of the invasive weed garlic mustard (Alliaria petiolata).

Sinha A. and S. Brault. 2005. Assessing sustainability of nontimber forest product extractions: how fire affects sustainability. Biodiversity and Conservation 14: 3537-3563.

Skellam, J. G. 1966. Seasonal periodicity in theoretical population ecology. Proceedings of the 5th Berkeley Symposium on Mathematical Statistics and Probability 4:179-205.

Smith, M., H. Caswell, and P. Mettler-Cherry. 2005. Stochastic flood and precipitation regimes and the population dynamics of a threatened floodplain plant. Ecological Applications 15:10361052 .

Spencer, M. and B. L. McGee. 2001. A field-based population model for the sediment toxicity test organism Leptocheirus plumulosus: I. Model development. Marine Environmental Research 51:327-345.

Steets, J. A., T. M. Knight, and T. L. Ashman. 2007. The interactive effects of herbivory and mixed mating for the population dynamics of Impatiens capensis. American Naturalist 170:113-127.

Stokes, K., A. Allchin, J. Bullock, and A. Watkinson. 2004. Population responses of Ulex shrubs to fire in a lowland heath community. Journal of Vegetation Science 15:505-514.

Strasser, C.A., M.G. Neubert, H. Caswell, and C.M. Hunter. 2010. Contributions of high- and low-quality patches to a metapopulation with stochastic disturbance. Theoretical Ecology doi 10.1007/s12080-010-0106-9.

van den Berg, F., C. A. Gilligan, J. C. Gerdessen, L. A. H. Gregoire, and F. van den Bosch. 2010. Optimal weed management in crop rotations: incorporating economics is crucial. Weed Research, 50:413-424. 
Vavrek, M. C., J. B. McGraw, and H. S. Yang. 1997. Within-population variation in demography of Taraxacum officinale: Season- and size-dependent survival, growth and reproduction. Ecology 85:2098-2107.

Vega, E. and C. Montana. 2004. Spatio-temporal variation in the demography of a bunch grass in a patchy semiarid environment. Plant Ecology 175:107-120.

Verdy, A. and H. Caswell. 2008. Sensitivity analysis of reactive ecological dynamics. Bulletin of Mathematical Biology 70:1634-1659.

Westerman, P., M. Liebman, F. D. Menalled, A. H. Heggenstaller, R. G. Hartzler, and P. M. Dixon. 2005. Are many little hammers effective? - Velvetleaf (Abutilon theophrasti) population dynamics in two- and four-year crop rotation systems. 53:382-392.

Wittmer, H. U., R. A. Powell, and C. M. King. 2007. Understanding contributions of cohort effects to growth rates of fluctuating populations. Journal of Animal Ecology 76:946-956.

Yoccoz, N. G., K. Nakata, N. C. Stenseth, and T. Saitoh. 1998. The demography of Clethrionomys rufocanus: from mathematical and statistical models to further field studies. Researches on Population Ecology 40:107-121. 


\section{A A brief survey of matrix calculus}

This appendix presents a brief survey of some of the basic manipulations involved in matrix calculus. The material is modified from Caswell (2008) under the terms of a Creative Commons Attribution Non-Commercial License. The paper in which the material originally appeared is available freely online at: http://www.demographic-research.org/volumes/vol18/3/

Matrix calculus permits the consistent differentiation of scalar-, vector-, and matrix-valued functions of scalar, vector, or matrix arguments. This appendix presents a brief statement of some essential results. More detail can be found in Caswell (2007, 2009, Klepac and Caswell 2010). A good introductory mathematical treatment is found in Abadir and Magnus (2005), and the most detailed presentation is the book of Magnus and Neudecker (1988).

There exist several conventions for matrix calculus, differing in their arrangements of the matrix and vector entries. The best is that of Magnus and Neudecker $(1985,1988)$.

If $x$ and $y$ are scalars, the derivative of $y$ with respect to $x$ is the familiar derivative $d y / d x$. If $\mathbf{y}$ is a $n \times 1$ vector and $x$ a scalar, the derivative of $\mathbf{y}$ with respect to $x$ is the $n \times 1$ vector

$$
\frac{d \mathbf{y}}{d x}=\left(\begin{array}{c}
\frac{d y_{1}}{d x} \\
\vdots \\
\frac{d y_{n}}{d x}
\end{array}\right)
$$

If $y$ is a scalar and $\mathbf{x}$ is a $m \times 1$ vector, the derivative of $y$ with respect to $\mathbf{x}$ is the $1 \times m$ gradient vector

$$
\frac{d y}{d \mathbf{x}^{\top}}=\left(\begin{array}{ccc}
\frac{\partial y}{\partial x_{1}} & \cdots & \frac{\partial y}{\partial x_{m}}
\end{array}\right)
$$

Note the orientation of $d \mathbf{y} / d x$ as a column vector and $d y / d \mathbf{x}^{\top}$ as a row vector; this reappears in the derivative of a vector with respect to another vector.

If $\mathbf{y}$ is a $n \times 1$ vector and $\mathbf{x}$ a $m \times 1$ vector, the derivative of $\mathbf{y}$ with respect to $\mathbf{x}$ is the $n \times m$ Jacobian matrix whose $(i, j)$ entry is

$$
\frac{d \mathbf{y}}{d \mathbf{x}^{\top}}=\left(\frac{d y_{i}}{d x_{j}}\right)
$$

Note that equations (78) and (79) are special cases where $m=1$ or $n=1$, respectively, and that if $m=n=1$ (i.e., the vectors are actually scalars) then (80) reduces to the usual scalar derivative.

Derivatives involving matrices are written by transforming the matrices into vectors using the vec operator (which stacks the columns of the matrix into a column vector), and then applying the rules for vector differentiation. Thus, the derivative of the $m \times n$ matrix $\mathbf{Y}$ with respect to the $p \times q$ matrix $\mathbf{X}$ is the $m n \times p q$ matrix

$$
\frac{d \operatorname{vec} \mathbf{Y}}{d \operatorname{vec}^{\top} \mathbf{X}}
$$

For notational convenience, we will write $\operatorname{vec}^{\top} \mathbf{X}$ for $(\operatorname{vec} \mathbf{X})^{\top}$.

These definitions (unlike some alternatives; see Magnus and Neudecker 1985) lead to the familiar chain rule. If $\mathbf{Y}$ is a function of $\mathbf{X}$ and $\mathbf{X}$ is a function of $\mathbf{Z}$, then

$$
\frac{d \operatorname{vec} \mathbf{Y}}{d \operatorname{vec}^{\top} \mathbf{Z}}=\frac{d \operatorname{vec} \mathbf{Y}}{d \operatorname{vec}^{\top} \mathbf{X}} \frac{d \operatorname{vec} \mathbf{X}}{d \operatorname{vec}^{\top} \mathbf{Z}} .
$$


The derivatives of matrices are constructed by forming the differentials of the expressions involving the matrices. The differential of a matrix (or vector) is the matrix (or vector) containing the differentials of the elements; i.e.,

$$
d \mathbf{X}=\left(d x_{i j}\right) .
$$

The key to the construction is the result that if, for vectors $\mathbf{x}$ and $\mathbf{y}$ and some matrix $\mathbf{Q}$, it can be shown that

$$
d \mathbf{y}=\mathbf{Q} d \mathbf{x}
$$

then

$$
\frac{d \mathbf{y}}{d \mathbf{x}^{\top}}=\mathbf{Q}
$$

(the "first identification theorem" of Magnus and Neudecker (1985)).

The combination of the chain rule and the identification theorem permits more complicated expressions involving differentials to be turned into derivatives with respect to an arbitrary vector, say $\mathbf{u}$. If

$$
d \mathbf{y}=\mathbf{Q} d \mathbf{x}+\mathbf{R} d \mathbf{z}
$$

then

$$
\frac{d \mathbf{y}}{d \mathbf{u}^{\top}}=\mathbf{Q} \frac{d \mathbf{x}}{d \mathbf{u}^{\top}}+\mathbf{R} \frac{d \mathbf{z}}{d \mathbf{u}^{\top}}
$$

for any $\mathbf{u}$.

The construction of the matrices makes extensive use the Kronecker product, defined as

$$
\mathbf{A} \otimes \mathbf{B}=\left(\begin{array}{ccc}
a_{11} \mathbf{B} & a_{12} \mathbf{B} & \cdots \\
a_{21} \mathbf{B} & a_{22} \mathbf{B} & \ldots \\
\vdots & \vdots & \ddots
\end{array}\right)
$$

The vec operator and the Kronecker product are related (Roth 1934); if

$$
\mathbf{Y}=\mathbf{A B C}
$$

then

$$
\operatorname{vec} \mathbf{Y}=\left(\mathbf{C}^{\top} \otimes \mathbf{A}\right) \operatorname{vec} \mathbf{B} .
$$

Thus the typical procedure for obtaining derivatives is

1. write a matrix expression involving the output variable,

2. take differentials of the expression,

3. apply the vec operator,

4. use Roth's theorem to an expression like (84) or (86), and

5. apply the First Identification Theorem to obtain the desired derivatives.

The chain rule can then be used to extend the result to obtain derivatives with respect to other variables. 


\section{Appendix citations}

Abadir, K. M. and J. R. Magnus. 2005. Matrix algebra. Cambridge University Press, New York, New York, USA.

Caswell, H. 2007. Sensitivity analysis of transient population dynamics. Ecology Letters 10:1-15.

Caswell, H. 2009b. Stage, age, and individual stochasticity in demography. Oikos 118:1763-1782.

Klepac, P. and H. Caswell. 2010. The stage-structured epidemic: linking disease and demography with a multi-state matrix approach model. Theoretical Ecology 4:301-319.

Magnus, J. R. and H. Neudecker. 1985. Matrix differential calculus with applications to simple, Hadamard, and Kronecker products. Journal of Mathematical Psychology 29:474-492.

Magnus, J.R. and H. Neudecker. 1988. Matrix differential calculus with applications in statistics and econometrics. John Wiley \& Sons, New York, New York, USA.

Roth, W.E. 1934. On direct product matrices. Bulletin of the American Mathematical Society 40:461-468. 